\title{
Clinical sacroiliac tests in ankylosing spondylitis and other causes of low back pain-2 studies
}

\author{
P. W. BLOWER AND A. J. GRIFFIN \\ From the Department of Rheumatology, Greenwich District Hospital, Vanbrugh Hill, London SE10 $9 H E$
}

SUMMARY Independent assessment by 2 observers of 4 tests for sacroiliac (SI) pain in patients with either mechanical/degenerative low back pain (M/D LBP) or ankylosing spondylitis (AS) showed all 4 to be reproducible, but only 2 of them, namely, pressure over the anterior superior iliac spines and pressure over the lower half of the sacrum, gave worthwhile discrimination. Positive results in these 2 tests were significantly associated with definite AS but also with the combination of low back pain, the HLA B27 antigen, and normal or near normal radiographs, a condition we have called presumptive ankylosing spondylitis.

Romanus and Ydén ${ }^{1}$ and Newton ${ }^{2}$ described how tests for sacroiliac (SI) pain were sometimes positive in early ankylosing spondylitis (AS) and might precede radiological changes by some years, but doubts about their value have remained. Some standard rheumatological texts ${ }^{3-5}$ mention that these tests may be positive in AS, but like the previous authors ${ }^{12}$ none cites their reproducibility, specificity, or sensitivity, and unlike the earlier authors none indicates the possibility of these tests being positive before radiological changes occur in the SI joints. Another standard text ${ }^{6}$ does not mention them at all, and a recent paper ${ }^{7}$ has claimed that such tests are of no value in distinguishing between AS and other forms of chronic low back pain (LBP).

In this paper we describe an interobserver study of 4 tests for SI pain between groups of patients with AS and mechanical/degenerative LBP(M/D LBP). Only 2 were found to be reproducible and significantly associated with AS, and these were employed in a prospective study, by one observer, of 500 adults who presented consecutively to a rheumatological clinic with a main complaint of LBP. Positive tests were significantly associated with definite AS, New York criteria, ${ }^{8}$ pregnancy, and with the possession of the HLA B27 antigen in the presence of normal or near normal radiographs, a condition we have called presumptive ankylosing spondylitis.

\section{Patients and methods}

In the first study 33 patients listed in the departmental index as having AS and 33 patients with LBP (pain

Accepted for publication 7 March 1983

Correspondence to Dr P. W. Blower felt between the 12 th rib and gluteal fold) of mechanical/degenerative origin were examined independently on the same day by both authors. The first examiner always knew the agreed diagnosis, but the second examiner did not know the diagnosis and performed only the tests described below before recording his/her findings. They used 4 tests for SI pain:

(1) Pressure on anterior superior iliac spines-patient supine.

(2) Forced adduction of the flexed thigh-patient supine.

(3) Lateral compression of the pelvis-patient on side.

(4) Pressure on the lower half of the sacrum-patient prone. Pressure was also applied over the lumbar spine with the patient prone. Tests for SI pain were considered positive only if the patient complained of pain directly over the sacrum and/or laterally in either or both buttocks. If pain was felt predominantly at the lumbosacral junction or above, the test was graded negative. If direct pressure over the sacrum and over the lumbar spine both produced pain, only the more painful test was graded positive. Standard AP and lateral radiographs of the lumbar spine were available for all patients. Only tests agreed as positive by both observers were used in the intergroup analysis.

In the second study the 2 tests for SI pain validated in the first, were performed by one of us (P.W.B.) as part of the routine physical examination of 500 Caucasians between the ages of 16 and 64 inclusive who presented consecutively to a rheumatology clinic over a 2-year period with a main complaint of LBP 
plus or minus leg pain. The very few cases of neoplastic disease during this period were not entered in the study.

Statistical analyses of both studies were done by the chi-square test with Yates's correction where applicable.

\section{Results}

All 5 tests showed excellent interobserver agreement (Table 1). Although forced adduction of the flexed thigh and pressure over the lumbar spine were the most frequently positive tests, neither had any discriminatory ability (Table 2 ). The 2 tests significantly associated with AS-pressure over the anterior superior iliac spine and sacral pressure-were not always both positive (anterior superior iliac spines alone $=2$, sacral pressure alone $=4$, both $=5$ ), indicating it is essential to perform both when screening for SI pain.

On reviewing the 33 patients in the AS group we found that only 28 satisfied the New York criteria ${ }^{8}$ for definite AS. The remaining 5 had been listed in the departmental index as having possible early AS. All 5 had normal radiographs, all had shown positive SI tests on more than one occasion in the past, 4 possessed the HLA B27 antigen, and the one B27 negative patient had psoriasis and a peripheral arthritis. Four of these still showed positive tests. Even if

Table 1 Interobserver reproducibility of tests in 66 patients

\begin{tabular}{llcl}
\hline Tests & Agreed & Disagreed & $p$ \\
\hline Ant. sup. iliac spines & 63 & 3 & $0 \cdot 001$ \\
Adduction of flexed thigh & 53 & 13 & $0 \cdot 001$ \\
Lateral compression & 64 & 2 & $0 \cdot 001$ \\
Sacral pressure & 64 & 2 & $0 \cdot 001$ \\
Lumbar pressure & 57 & 9 & $0 \cdot 001$ \\
\hline
\end{tabular}

Table 2 Specificity of tests in 66 patients

\begin{tabular}{lccl}
\hline Agreed positive tests & 33 AS & 33 LBP & $p$ \\
\hline Ant. sup. iliac spines & 7 & 0 & 0.05 \\
Adduction of flexed thigh & 5 & 13 & NS \\
Lateral compression & 0 & 0 & NS \\
Sacral pressure & 9 & 0 & 0.01 \\
Lumbar pressure & 11 & 23 & NS \\
\hline
\end{tabular}

Table 3 SI tests in 500 new patients with LBP

\begin{tabular}{lrr}
\hline & $L B P$ & Definite $A S$ \\
\hline$(+)$ & 9 & $8 \quad(\mathrm{p}<0 \cdot 001)$ \\
$(-)$ & 471 & 12 \\
Total & 480 & 20 \\
\hline
\end{tabular}

these 5 are removed from the analysis, the difference between the groups remains significant (positive tests in $7 / 28 \mathrm{AS}$ patients and $0 / 33 \mathrm{M} / \mathrm{D}$ LBP patients, $\left.\chi^{2}=7 \cdot 02, \mathrm{p}<0 \cdot 01\right)$.

In the second study 9 of the 17 patients with positive SI tests had normal or near normal radiographs (two had grade II changes ${ }^{8}$ in one SI joint (Table 3 ). Four of these possessed the B27 antigen, and, if it is assumed that the 480 patients without definite AS possessed an average spectrum of tissue types and the prevalence of HLA B27 was normal at $8 \%$, then the association of positive tests with the presence of B27 in these patients is highly significant $\left(\chi^{2}=10 \cdot 43\right.$, $\mathrm{p}<0.002)$.

Of the 5 with positive tests who did not possess B27 2 were pregnant, one of whom had psoriasis. Both were $x$-rayed after the birth of their children, and the one without psoriasis had unilateral osteiitis condensans ilii. A third patient was 6 weeks post partum. In this survey there were 124 women under the age of 41 who had neither definite nor presumptive AS, but these were the only 3 who were pregnant or puerperal, and we think it likely that the positive tests are related to the hormonally induced ligamentous laxity which occurs towards the end of pregnancy and in the puerperium and which permits increased SI joint movement. The association is again highly significant $\left(\chi^{2}=63 \cdot 20, p<0 \cdot 001\right)$. One of the remaining 2 had a 10 -year history of intermittent right buttock pain, grade II radiological changes ${ }^{8}$ in the right SI joint, and a subsequent clinical pattern much more like low-grade AS than M/D LBP. The other patient was clinically normal when seen 4 weeks later.

Although the 4 people with LBP, positive SI tests, HLA B27 and normal or near normal radiographs do not satisfy either the New York ${ }^{8}$ or Rome ${ }^{9}$ criteria for $^{2}$ AS, we feel that these 4 patients are probably suffering from an early or mild form of AS which could be described by the phrase 'presumptive ankylosing spondylitis'. This hypothesis is supported by: (1) both studies show a significant relationship between positive SI tests and definite $\mathrm{AS}^{8} ;(2)$ the second study shows a significant relationship between positive SI tests and the possession of HLA B27 in the presence of normal or near normal radiographs; (3) HLA B27 is closely associated with definite AS in Caucasians. ${ }^{1011}$

We also consider that the 5 patients in the AS group of the first study who had LBP, normal radiographs, and positive SI tests on at least 2 occasions should be similarly classified. The main features of these 9 patients are listed in Table 4 , the key point being that in this group positive SI tests were the only sign in the musculoskeletal system pointing towards a diagnosis of AS. 
Table 4 Nine patients with presumptive ankylosing spondylitis

\begin{tabular}{ll}
\hline SI tests $(+)$ & 9 \\
B27 $(+)$ & 8 \\
B27 $(-$ ), psoriasis & 1 \\
All lumbar movements limited & 0 \\
Chest expansion $=2 \cdot 5 \mathrm{~cm}$ or less & 0 \\
ESR (Westergren) $>15 \mathrm{~mm} / \mathrm{h}$ & 1
\end{tabular}

Men:women=2:7.

Mean age of onset $=23(18-28)$ years.

Onset to diagnosis (median) $=12$ months ( 6 months -12 years)

\section{Discussion}

The importance of these tests, which in both studies show low sensitivity but high specificity, is not in their application to patients who have definite AS. $^{8}$ Although it is satisfying to the clinician correctly to diagnose AS in a patient presenting with back pain, solely on the history and physical signs, nearly all such patients will have radiographs of the lumbar spine performed. If these show bilateral grade III/IV sacroiliitis, ${ }^{8}$ the New York criteria ${ }^{8}$ for definite AS are fulfilled. In such patients the physical signs of generalised limitation of lumbar movements (the New York criteria ${ }^{8}$ incorrectly describe the movements of lumbar flexion, extension, and lateral flexion as occurring in 3 planes, but the movements of flexion and extension are merely different arcs of the same plane - the sagittal) and/or limitation of chest expansion may suggest the correct diagnosis to the clinician and indicate the distribution and severity of the disease, but they are not necessary for defining it. If the radiological signs are less, then either may be essential to fulfil the criteria for definite AS. ${ }^{8}$ The real value of SI tests is their ability to pick out one presentation, sacroiliitis, of early or mild AS. Whether all such patients will ultimately develop definite $A S^{8}$ is unknown, but it would seem possible that some cases of presumptive ankylosing spondylitis may remain formes frustes of AS and never satisfy the current agreed criteria. ${ }^{89}$ (Two women gave histories of buttock pain for 10 and 12 years respectively and both showed only unilateral grade II radiographic changes ${ }^{8}$ in the SI joints.)

Why both these studies showed that positive results in 2 of the tests were significantly associated with definite AS, ${ }^{8}$ and the recent study by Russell $e t$ $a l .{ }^{7}$ suggested that these same tests had no ability to discriminate between AS and other forms of LBP, is almost certainly due to differences in defining what constituted a positive response. Russell et al. ${ }^{7}$ appear to have graded any painful response as positive regardless of where the pain was produced, whereas in both these studies as described under 'Patients and methods' SI tests were graded positive only if pain was produced over the sacrum and/or laterally in either or both buttocks. It was not uncommon in both our studies for pressure over the lower half of the sacrum or pressure over the anterior superior iliac spines to cause pain in the lumbosacral region or above in people apparently suffering from M/D LBP. This is not unexpected, in that sudden pressure over the lower half of the sacrum or over the anterior superior iliac spines with the patient on a couch possessing some springiness may produce movement of the sacrum relative to the lumbar spine and thus cause pain in a mechanically disordered or degenerative lumbosacral or lumbar joint. That none of the 9 patients with presumptive ankylosing spondylitis had a chest expansion of $2.5 \mathrm{~cm}$ or less is not surprising, as this degree of limitation is a late manifestation of AS. ${ }^{612}$ That none had generalised limitation of lumbar movements is perhaps less expected, but this too takes time to develop, and 6 of these patients had had symptoms for 15 months or less.

Both studies suggest that pain produced by stressing the SI joints usually indicates an inflammatory sacroiliitis and that mechanical-degenerative disorders of the SI joints, 'sacroiliac strain', are an uncommon cause of LBP unless the patient is pregnant or puerpural.

\section{Conclusions}

All 4 tests for SI pain that we studied showed good interobserver agreement, but only 2-pressure over the anterior superior iliac spines and pressure over the lower half of the sacrum-were significantly associated with definite AS. ${ }^{8}$ Positive tests were also significantly associated with the combination of LBP, the HLA B27 antigen, and normal or near normal radiographs, a condition we have called presumptive ankylosing spondylitis. They were also significantly associated with pregnancy.

\section{References}

1 Romanus R, Ydén S. Pelvo-spondylitis ossificans. Copenhagen: Munksgaard, 1955: 18-23.

2 Newton D R L. Discussion on the clinical and radiological aspects of sacro-iliac disease. Proc $R$ Soc Med 1957; 50: 850-3.

3 Moll J M H. Ankylosing spondylitis. In: Scott J T, ed. Copeman's textbook of the rheumatic diseases. Edinburgh, London, New York: Churchill Livingstone, 1978: 511-36.

4 Boyle J A, Buchanan W W. Clinical rheumatology. Oxford: Blackwell, 1971: 284.

5 Bluestone R. Ankylosing spondylitis, In: McCarty D J, ed Arthritis and allied conditions. 9th ed. Philadelphia: Lea and Febiger, 1979: 620-32.

6 Calin A. Ankylosing spondylitis. In: Kelley W N, Harris E D, Ruddy S, Sledge C B, eds. Textbook of rheumatology. Philadelphia: Saunders, 1981: 1017-32.

7 Russell A S, Maksymowych W, LeClerc S. Clinical examination of the sacroiliac joints: a prospective study. Arthritis Rheum 1981; 24: 1575-81.

8 Bennett $\mathrm{P} \mathrm{H}$, Wood $\mathrm{P} \mathrm{N} \mathrm{H}$. Third international symposium on population studies in the rheumatic diseases. Amsterdam: Excerpta Medica, 1980; 148: 456-7. 
9 Kellgren J H. Diagnostic criteria for population studies. Bull Rheum Dis 1962; 13: 291-2.

10 Brewerton D H, Caffrey M, Hart F D, James D C O, Nicholls A, Sturrock R D. Ankylosing spondylitis and HL A27. Lancet 1973; i: 904-7.
11 Schlosstein L, Terasaki P I, Bluestone R, Pearson C M. High association of HL-A antigen, W27 with ankylosing spondylitis. N Engl J Med 1973; 288: 704-6.

12 Moll J M H, Wright V. New York clinical criteria for ankylosing :spondylitis. Ann Rheum Dis 1973; 32: 354-63. 